\title{
Payload motion control of rotary gantry and luffing cranes using mechanical wave concepts
}

\author{
Hossein Habibi ${ }^{a *}$, William O'Connor ${ }^{\text {b }}$ \\ ${ }^{\text {a }}$ Department of Mechanical Engineeing, Persian Gulf University, Bushehr 75168, Iran \\ ${ }^{\mathrm{b}}$ UCD School of Mechanical and Materials Engineering, Engineering Building, UCD Belfield, Dublin 4, \\ Ireland.
}

\begin{abstract}
A new solution is presented to the problem of controlling the motion of a crane's suspended load through arbitrarily-complex, 3-D paths through the crane's manoeuvre space. A generalised boom crane arrangement is considered, so that gantry and luffing arrangements are included as particular cases. Thus the crane's boom slews about a central, vertical (tower) axis. This boom is either a horizontal gantry with a trolley moving radially along it, from which the load can be winched; or a jib which can rotate in the vertical plane, with the hoisting cable passing over a pulley attached at its end point. In either case there are three directly controlled motion variables, whose effects on the suspended payload's motion are strongly cross-coupled. The challenge is to get the payload to follow the desired 3-D path as closely as possible during the manoeuvre, and come to rest rapidly at target, by directly controlling these three actuating motions. Thus the controller must achieve position control combined with active swing suppression throughout the manoeuvre and on arrival at the desired end point. A model is developed of the generalised crane for both gantry and luffing crane types. The proposed control strategy is then applied and tested on this model. The controller is based on mechanical wave concepts. When applied to the model it is shown to be very effective. It is accurate, robust to system changes and actuator limitations, very stable, requires sensing only at the trolley (and not at payload), and is easy to implement.
\end{abstract}

\section{Keywords:}

Wave based control, tower (rotary) crane, boom crane, luffing crane, hoisting

\footnotetext{
* Corresponding author: e-mail: habibi@pgu.ac.ir
} 


\section{Introduction}

Cranes to lift heavy loads are ubiquitous, on building sites, in ports, in factories and workshops, on ships, in airports, in storehouses and at truck depots. After the load-carrying capacity and operational workspace have been considered, the crane's dynamic performance can be defined in many ways, but aspects such as throughput, final positioning accuracy, transit speed, obstacle avoidance, and minimising sway during and after transit, can all be important (Abdel-Rahman et al., 2003). There are many crane designs in wide use. Most cranes are under human control, often demanding high levels of skill. Increasingly however cranes have control assistance for the driver and sometimes they can be totally automated. This paper is intended for total automation, in which the payload is intended to move from rest to rest along a specified, arbitrarily-complex path in 3-D, limited only by the crane's operational space.

A Cartesian gantry crane, in which the overhead trolley moves along a moving rail, allowing the trolley to be moved horizontally in two perpendicular directions (typically found in factories, for example) is relatively easy to control. The payload position control and cable swing control can be treated as if uncoupled, especially for smaller swing angles, allowing almost independent control of each axis. Each Cartesian axis can be treated as a single axis control problem, for which various solutions are available. On the other hand, cranes which have a central tower supporting a rotating boom (often found on construction sites for example) pose a greater motion control challenge due to the strong coupling between tangential and radial motions, even without considering hoisting effects. Cranes with luffing action, where the main boom also rotates in the vertical plane to lift the load, are generally even more challenging, with even stronger cross-coupling between the effects of actuation motion on the load motion, affecting the gross motion and the load swing motion.

This paper considers the control of such rotary tower cranes, both the horizontal-gantry and vertically luffing kinds. In both cases there are three directly controlled variables. In the first case there is the slewing motion, (rotation about tower axis), the trolley motion along the beam (radial 
distance with respect to the tower), and the hoisting motion. In the second case the three motions variables are the slewing angle, the luffing angle of the boom and the hoisting cable length. The challenge is how to use these directly controlled motions to control, indirectly, the motion of the suspended load, combining position control (path following) and active swing damping.(So called "level-luffing" cranes have special provision to keep the load at constant height during luffing, thereby removing some of the coupling between motion components, but leaving radial and rotational motions still coupled.)

Even with no external disturbance, some pendular motion is inevitable. As manoeuvre speeds (or accelerations) increase the pendulation during and after transits can become very significant. The coupling effects between the slewing, luffing and hoisting motions also increase with increased accelerations. Cranes are inherently lightly damped (Tedd et al., 1997), and the load inertias can be very large. Safety, speed and efficiency demand that the load be well controlled, and in a way that is robust to a wide range of cable lengths, payloads, and other parameter uncertainties. Gustafsson (1995) and Abdel-Rahman and Nayfeh (2001) have shown that for a boom crane a single planar controller cannot stabilize payload oscillations, and so control should be applied both in-plane and out-of-plane to cope with payload pendulation. It also has been found that if a control system for perfect suppression of swinging is needed, it is necessary to use a variety of specially constructed add-on actuators, in addition to the boom's slewing and luffing actuators (Abdel-Rahman et al., 2003). This approach, however, seems to add considerable expense and complexity.

In contrast with the many papers on Cartesian gantry cranes, there are few rotary or boom crane control strategies reported in the literature. Standard control techniques are generally used for rotary and boom cranes, either closed-loop or open-loop, but these techniques have challenges such as the development of payload pendulation along the path which increases as the slewing angle increases (Sakawa et al., 1981; Takeuchi et al., 1988). Recently successful anti-sway control for rotary cranes has been reported. For example, Neupert et al. (2010) introduce a practical solution for tracking and 
anti-sway control of boom cranes based on differential flatness. They show impressive progress in achieving sway-free load transits. The decentralized control, however, is based on exact linearization. Moreover all rope angles and angular velocities must be available, so gyroscopes are needed to measure angular velocities in both tangential and radial directions. Then two observers using gyroscope data are required to eliminate natural, higher-order frequencies of the rope. So these observers have to be adapted to the system dynamics, and the determined eigenvalues depend on parameters such as the load dynamics and cable length.

In a more recent study, an open-loop controller to suppress load sway in rotary cranes was presented using only horizontal boom motion, and seems to be energy efficient and safe (Uchiyama et al., 2013). Although the developed approach removes the need to measure the load sway it copes only with a simple model of rotary crane dynamics, compared with the current work that deals with 3D rotary boom crane moving a payload to track complicated spatial trajectories. In another similar work presented recently by Matuško et al. (2015), the proposed feedback controller can perform positioning and swing minimization of heavy loads for 3D tower cranes. However the control design is based on a tensor product model transformation with an additional neural network based friction compensator. This makes the control performance dependent on having an accurate system model, unlike the approach presented here.

Blajer and Kołodziejczyk (2007), (2011) have looked at the problem from the perspective of multibody dynamics in which the effects of the actuators are considered as "servo-constraints" on the system (as distinct from natural and geometric constraints). The direction of the control forces may be tangent to the servo-constraint manifold, leading to an interesting control challenge. The governing differential-algebraic equations are transformed by projecting them onto the orthogonal and tangent subspaces with respect to the servo-constraint manifold in the crane velocity space. The control system is made more robust by incorporating feedback of the load position error. 
By contrast, the control strategy to be presented below is not dependent on having a perfect, or even good, system model, or system equations of motion, nor on knowing the dynamic system parameters such as the load dynamics and cable length. For this reason, the controller needs little sensing which has financial and reliability benefits: improved performance without extra hardware. Probably the largest economic benefit comes from achieving very good trajectory following without the need for highly skilled crane-drivers (or indeed any human drivers), or other human operators on the ground guiding the load with guylines. The proposed wave-based control (WBC) method requires knowledge only of the directly controlled crane motion variables (which generally will be easily available from the actuator controllers) together with some measure of the horizontal forces where the cable meets the pulley or trolley (or even simply some measure of the cable's angular displacement from the vertical). When the desired payload path through space, in three dimensions, has been specified, it implicitly specifies a combination of values for the three, directly-controlled, input variables which, if inertial, pendulation and other dynamic effects could be eliminated, would cause the hanging load to follow the desired 3-D spatial curve exactly. Determining these inputs is a purely kinematic task, based on the crane's geometry, with its fixed and variable lengths and angles. In the proposed control strategy, these three variables over time determine one component of the reference inputs to the motion actuators. The second components are determined as described below and are designed to achieve active swing control.

In Section 2, a model of a generalised rotary-boom crane will be presented, in such a way that the gantry and luffing versions become special cases. In Section 3, an introduction to WBC and its significant adaptation to this problem are presented. Section 4 shows the results of applying the modified WBC to control the load using the different rotary crane types. Section 5 is a summary and conclusion. 


\section{Modeling}

Figure 1 depicts the generalised crane model. Real cranes generally will not have all the degrees of freedom shown. This configuration however combines in one model most ways rotary tower cranes are configured, whether gantry of luffing. Real crane designs will correspond to restricted versions of this model, as special cases. The two most important special cases will now be considered.

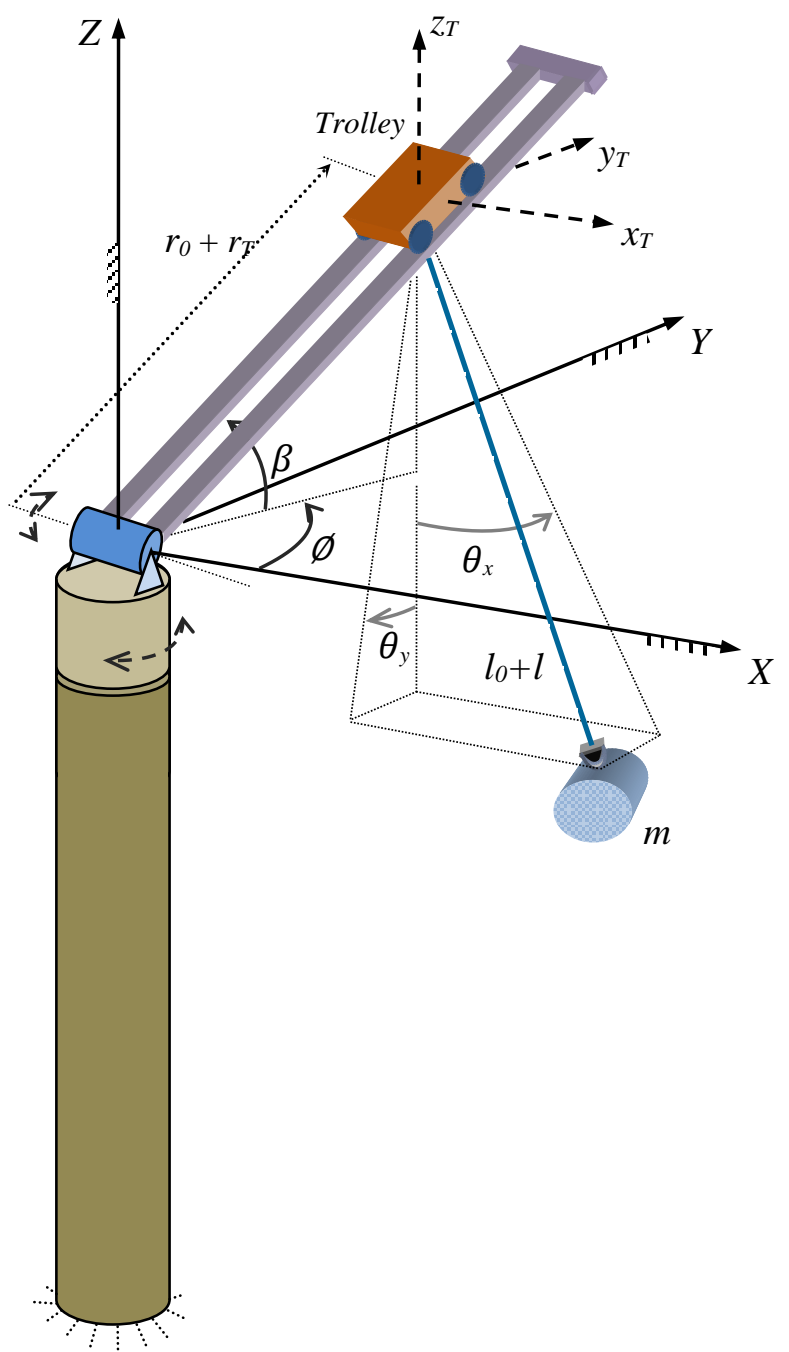

Fig. 1: A general representation of the 3D model of rotary/boom crane

\subsection{Tower (rotary) gantry crane model}

As the first option, the model shown in Fig.1 can be simplified to a tower (rotary) crane in which the luffing angle $\beta$ is assumed to be constant at zero $(\beta=0)$. Then the girder (jib), illustrated with a 
slotted bar over which trolley translates, remains horizontal and rotates only in the $(X Y)$ plane, about a fixed vertical $Z$-axis. This allows the trolley to have two motion components in the $X Y$-plane, a radial translation along the slotted jib and a rotation $(\varnothing)$ about the $Z$-axis. In addition, the hoisting of the load is modelled by varying the length of the cable. The constant values $r_{0}$ and $l_{0}$ shown in Fig. 1 represent the initial or minimal radial distance of the trolley from the tower and the initial or minimal length of the cable respectively. Figure 2 shows $(a)$ a plan view and $(b)$ a side view of the rotary gantry crane.

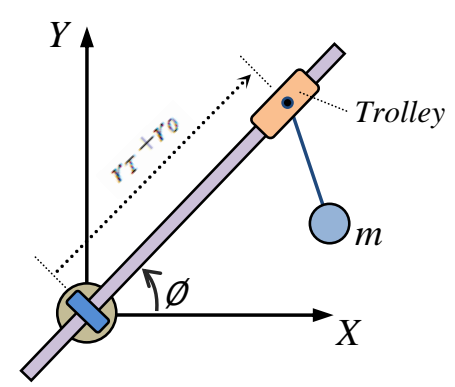

(a)

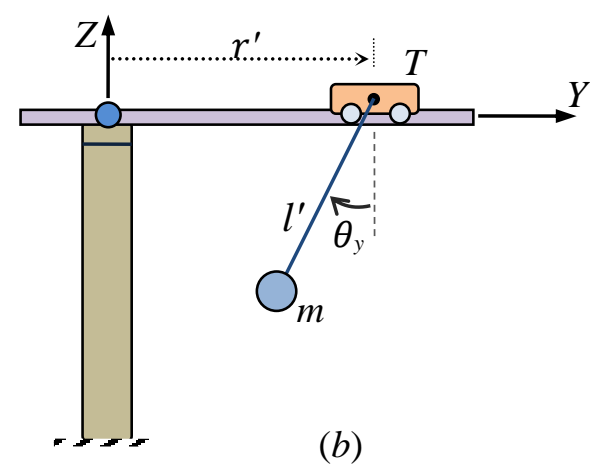

Fig. 2: Two views of the tower crane with hanging payload $a$ ) top view, b) side view seen along the $X$ axis

In Fig. $2(a)$, the only angle coordinate that is visible and accurately depicted is planar rotation $(\varnothing)$. Since there is no luffing action, the radial distance $r_{T}$ appears in the $X Y$-plane with the correct magnitude. On the other hand, in the view in Fig. $2(b)$, only the angle $\theta_{y}$ as shown corresponds to its actual value. On the other hand $l^{\prime}$ is the projected length of the cable in the $Y Z$-plane, defined as $l^{\prime}=\left(l+l_{0}\right) \cos \theta_{x}$; and $r^{\prime}$ is the radial distance of the trolley from the tower projected in the $Y$ direction, given by $r^{\prime}=\left(r_{T}+r_{0}\right) \sin \emptyset$. The gantry and trolley motions are therefore restricted to the horizontal $(X Y)$ plane, with no motion in the vertical $(Z)$ direction. So in this model, the inputs to the system are $r, \varnothing$ and $l$, where $l$ determines the hoisting of the payload.

\subsection{Luffing boom crane model}


The second special case allows the boom to rotate in the vertical as well as horizontal planes, and so is closer to the case depicted in Fig.1, with none of the rotational motions constrained. The boom rotates $\varnothing$ about the vertical $Z$-axis as before, whereas the luffing angle $\beta$ is about a horizontal axis. Figure 3 shows a side view with $\varnothing=0^{\circ}$ so that the luffing is in the $X Z$-plane. The moving trolley is then omitted, and the distance $r_{0}$ along the boom is fixed at the boom length.

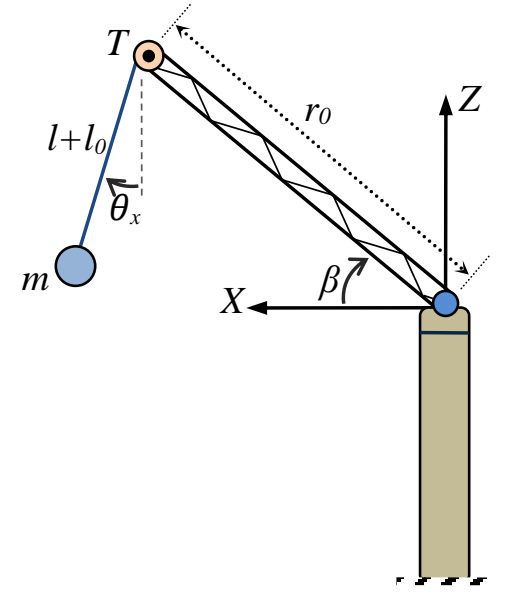

Fig.3: Side plan view from a boom crane considering no horizontal rotation $(\varnothing=0)$

The payload is raised using either luffing or cable hoisting, or a combination of the two. The crane model therefore has three controlled input variables: $\varnothing, \beta$ and $l$. The luffing motion of the boom can be achieved in practice in different ways, such as by a hydraulic cylinder mounted between the tower and the boom, or by guylines and winding cables. However, for the dynamic model and the control strategy the only relevant question is how the angle $\beta$ varies over time, regardless of the mechanisms used to achieve it in a given crane.

\subsection{Dynamic model}

A mathematical model of a three-dimensional rotary (boom) crane is now developed which includes both the gantry (or radial) motion and the luffing motion. Typical spherical load oscillations, which are excited by the crane's slewing and luffing motions, are described by the cable angles projected onto the $X Z$ and $Y Z$ planes, shown in Fig.1 as $\theta_{x}$ and $\theta_{y}$ respectively. Thus for a given cable length, the dynamic behavior of the payload has two degrees of freedom described by the two angles 
$\theta_{x}$ and $\theta_{y}$. In addition there are four input variables $(r, \varnothing, \beta$ and $l)$. A dynamic model of the load pendulation was derived using the Lagrange equations of the second kind (Euler-Lagrange equations). Due to the complexity of the resulting model, the Lagrange equations can be solved only numerically. A $4^{\text {th }}$ order Runge-Kutta integration method was used. Once developed, the dynamic responses of the crane models, without control, were tested to verify the model itself. The trolley was moved through simple paths causing the expected swaying response in the motion of the payload at the expected frequencies for different parameter values.

Having confirmed the dynamic response of the generalised crane model without control, the crane control design was then developed and tested. The next section gives a summary of the development of the new control strategy, including some challenges and early difficulties, along with the final successful strategy and implementation.

While respecting the main dynamic complexities, some simplifying assumptions were invoked to keep the equations of motion and the resulting crane model more manageable. These assumptions are as follows.

- As mentioned, the crane structure, comprising tower, girder and/or boom, is regarded as rigid.

- The load is modelled as a point mass.

- The distributed mass of the cable is negligible in comparison with the weight of the payload.

- The cable's longitudinal stiffness is very high and the flexural stiffness very low.

- External disturbing forces (other than gravity), such as wind loading, are negligible.

Note that these assumptions, commonly made in other studies, are here made for modelling purposes only, to capture the main dynamic features and interaction between control variables, without unnecessary detail. It transpires that the ultimate control scheme does not require many of these assumptions and it remains robust to many departures from such idealised model behaviour. For example, if the load is not well modelled by a point mass, but has more complex passive 
dynamics, it has been found that the same control strategy still performs well, with very good active sway control and zero final position error.

Even with these assumptions, the model takes full account of the coupling between the radial and tangential load sway when there is slewing, luffing, and radial movement. In many crane simulations the cable length $(l)$ is taken as constant, implicitly assuming slow hoisting speeds, whereas, in the current work, the first and second derivatives of hoisting will be involved in the equations of motion. Moreover the cable sway angles are not assumed to be small, with no small-angle, line arising assumptions.

\section{Adapted wave-based control}

As already explained, from a control viewpoint, the trolley (or cable suspension point) is considered to be a single, motion-controlled actuator, attempting to control an attached flexible system of non-trivial dynamics. In the generalised crane model, the system has a total of six degrees of freedom. In the generic crane model there are four directly controlled variables which determine the trolley's position in 3-D space, as well as the cable length. (In each of the two special cases, gantry and luffing, there are only three directly controlled variables, as one of the four is fixed). There are then two further degrees of freedom corresponding to the two cable swing angles. The output is the payload motion. It must be controlled to follow a desired trajectory. Clearly the four (or three) actuating variables do not act directly on the load motion, but must work through the intervening flexible dynamics to control the load's 3-D pendular motion, to follow the desired track. In this sense, the system is "under-actuated".

Classical feedback control strategies, such as PID, do not work well with such a system, mainly because of the inherent delays ("soft" interconnection) between actuator motion and output motion. Open loop techniques, such as command shaping or time-delay filtering, do respect the inherent delays, but require a good system model. As the order of the system grows, perhaps with distributed 
load mass, or multiple masses, it becomes more and more difficult to model and so to design the open-loop input, and the absence of feedback means any residual sway is not automatically corrected.

The proposed control strategy is a particular application of a more general technique for control of under-actuated, passive, flexible systems, called wave-based control. For more background on this approach the reader is referred to references (O'Connor, 2003, 2007; O'Connor et al., 2009). What follows is a summary introduction, assuming a single actuator controlling an attached flexible system.

This technique does not depend on having an accurate system model. It uses feedback, but the feedback measurements are taken not at the system output (here, the load position) but at the actuator (the trolley or boom tip pulley). In WBC, any motion of an actuator attached to a flexible system is seen as launching a mechanical wave (mechanical disturbance) from the actuator into the system, while responding to a mechanical wave returning from the system to the actuator, either to absorb it, reflect it, or, in general, to partly absorb and partly reflect it back into the system. In addition to oscillatory (AC) components (corresponding, in the case of a crane, to swaying motion), the propagating disturbance will also have a net (or DC) displacement component, which could be considered so-called rigid-body motion. For rest-to-rest manoeuvres to a target displacement, the wave enters the system from the actuator and later leaves through the same actuator, in such a way that the net displacement left behind is the target (DC) displacement, while the oscillations (AC) aspects are also totally absorbed. All sensing and control is done at the interface between the actuator and the flexible system, where energy and momentum are transferred into and out of the flexible system.

In the crane application of WBC considered here, the "flexible system" is considered to begin at the suspension point at the top of the hanging cable, and includes the deployed cable and attached load. Thus the load is considered part of the flexible system (whether the load is lumped, distributed, 
rigid or flexible). The "actuator", controlling this flexible system, is taken as the crane structure up to the cable suspension point, that is, the point where the cable leaves the trolley or pulley. The motion of this point is directly controlled by the crane actuators. It is assumed that the crane structure up to that point can be taken as rigid (certainly in comparison with the flexibility of the hanging cable and load) and can be controlled directly and precisely. The control problem is then seen as indirectly controlling the load's motion by directly controlling this suspension point's motion.

From the perspective of WBC, the "wave" can be understood as a disturbing motion, initiated by "the actuator", that is, the motion of the point at the top of the cable. The motion then propagates through the flexible system, that is, down the cable down to the load, and then back up to the actuator for reflection/absorption, with this motion happening repeatedly and continuously, dispersing as it goes, for as long as the motion continues. In this work the wave variable will be taken as translational displacement in 3-D space, seen as propagating along the hanging cable and within the load. In this view, the real motion of the load is considered as the superposition of notional component wave motions, in a way to be described below.

WBC has already been successfully applied to the gantry crane control problem, for a 1-D gantry system (O’Connor, 2003), and for a 3D double-pendulum gantry system with a 2-D Cartesian trolley (O’Connor and Habibi, 2013), both without hoisting. The main novelty here is that the technique has now been adapted for motion of a hanging load along an arbitrary path in 3-D space controlled by rotary-boom cranes, with hoisting also taken into account. Other novel aspects will also be presented below.

WBC uses the measured, mechanical-motion wave coming back to the actuator from the flexible system, the so called returning wave, to accomplish two main jobs, namely position control and active damping of the swing. The controller sets the input request to the trolley position (or cable suspension point) to be the sum of two components. The first is half the desired (reference or target) 
load displacement over time, referred to below as the launch wave, denoted $a$. The second is the returning wave, denoted $b$. The addition of this second component provides active damping of the sway motion while moving the system a net amount corresponding to the remaining half of the target position. Further details regarding the performance and efficiency of the WBC can be found in the literature (O’Connor, 2003, 2007; O’Connor et al., 2009).

In previous applications of WBC to systems moving in 2-D and 3-D, the control in each dimension (direction) was applied separately, in parallel, as if these motions were uncoupled. So there were separate 1-D wave-based control systems (launching and absorbing) in each direction, acting simultaneously. This worked very well. When however this approach was applied to the tower crane, separately controlling, for example, the radial and tangential components of the motion, the resulting control system was stable and achieved good swing control, but it usually resulted in final position errors for rest-to-rest motions. The crane load did not end up exactly at the reference position. Much work was dedicated to finding a way to modify the standard WBC strategy to eliminate these final, steady-state errors. It was clear that the errors arose from the coupling between the motion components, so that, for example, in absorbing the returning radial motion, some of the tangential motion was also being absorbed, and vice versa, leading to errors in the final values of both. But no satisfactory, generic way was found to deal with this problem, or to decouple the control of the motions: at least no way which also retained the main attractive features of WBC (robustness, minimal sensing, not model-based, simplicity, co-location of sensors and actuators, etc.).

Eventually a simple solution emerged which in retrospect seems almost obvious. Ultimately the cause of the cross-talk is associated with the coupled nature of the motions when viewed in the crane coordinate system. Momentum equations in Cartesian directions can be considered uncoupled. So instead of measuring and controlling the waves in crane coordinates, one could use Cartesian coordinates, not just to define the desired load trajectory (where they are often a natural choice), but also to achieve the launching and absorbing of the component waves. The crane control variables can 
be transformed into Cartesian variables, and the WBC implemented in the Cartesian coordinates. The definitions of the waves (see Eq.(1) below) can then be based on linear momenta in two orthogonal directions, $x$ and $y$, which are sufficiently uncoupled to ensure that the net change in momentum, in both directions, for a rest-to-rest manoeuvre, is zero. Furthermore, the system is so stiff in the vertical, or $z$ direction, that special control strategies are not necessary for motion components in this direction. The sway control can and should be achieved by suitably controlling the motion in the two horizontal directions, $x$ and $y$, while the desired hoisting can be directly applied without needing WBC.

Thus three Cartesian coordinates, $x_{r e f}, y_{r e f}, z_{r e f}$, each given as a function of time, define a desired load path over time in 3-D space. As discussed, the point where the cable meets the crane pulley or trolley is considered as the interface between the actuation point (whose motion, $x_{T}, y_{T}, z_{T}$, is directly controlled, taken as part of an assumed rigid body) and the flexible mechanical system (the swaying suspended system and load, indirectly controlled, and also affected by changes in the cable length due to hoisting). This is the point where the motion wave is considered to be launched from the rigid system into the flexible system and also where the returning wave is measured and re-absorbed. The returning waves which the actuator should absorb are defined as

$$
\begin{aligned}
& b_{x}=\frac{1}{2}\left[x_{T}-\frac{1}{Z_{x}} \int_{0}^{t} f_{x} d t\right] \\
& b_{y}=\frac{1}{2}\left[y_{T}-\frac{1}{Z_{y}} \int_{0}^{t} f_{y} d t\right]
\end{aligned}
$$

These expressions involve the horizontal Cartesian components of the actual trolley motion, $x_{\mathrm{T}}$ and $y_{T}$, and an integral of the component of the cable force in the $x$ and $y$ directions, divided by constant mechanical impedances $Z_{x}$ and $Z_{y}$. The choice of impedance values will be considered below. 


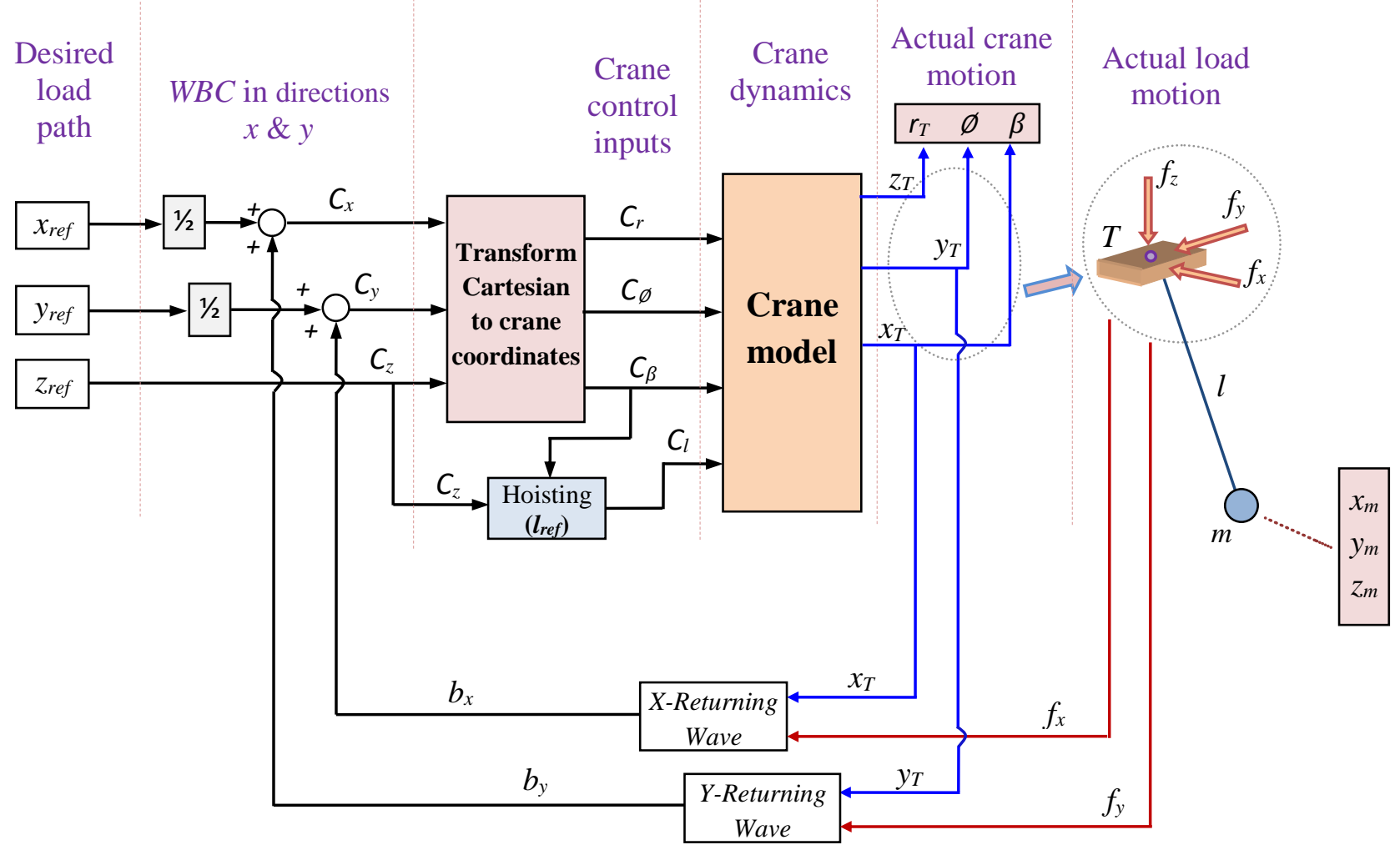

Fig. 4: WBC scheme for rotary crane model with hoisting

In practice, the controller sets the launch wave to be half the desired, or reference motion $\left(x_{r e f}, y_{r e f}\right)$ of the load, in 3-D space, over time. Simultaneously it measures the returning waves and adds these to the launch waves to determine the desired displacements so that

$$
\begin{aligned}
& C_{x}=\frac{1}{2} x_{r e f}+b_{x} \\
& C_{y}=\frac{1}{2} y_{r e f}+b_{y} \quad(a)
\end{aligned}
$$

The Cartesian coordinates of the load mass, $x_{m}, y_{m}, z_{m}$, are related to the directly controlled crane variables $r, \varnothing, \beta$ and $l$, as follows

$$
\left\{\begin{array}{l}
x_{m}=\left(r_{T}+r_{0}\right) \cos \beta \cos \emptyset+l \sin \theta_{x} \cos \emptyset \\
y_{m}=\left(r_{T}+r_{0}\right) \cos \beta \sin \emptyset-l\left\{\cos \theta_{x} \sin \theta_{y} \cos \emptyset+\sin \theta_{x} \sin \emptyset\right\} \\
z_{m}=\left(r_{T}+r_{0}\right) \sin \beta-l \cos \theta_{x} \cos \theta_{y}
\end{array}\right.
$$

The reference motion in the horizontal plane of the cable suspension point (that is, the trolley position for the gantry crane, the boom pulley position for the luffing crane) is identical to the 
horizontal components of the desired load motion, which is $x_{r e f}$ and $y_{r e f}$. In other words, if there was no sway, or if the entire crane system was rigid, the horizontal motion of the cable suspension point would be the same as the horizontal components of the target displacement of the load.

Regarding controlling the vertical or $z$ motion of the load, the situation is simplest for the gantry crane case, where the reference cable length at every instant is simply the negative of the target $z$ at that instant, or $-z_{\text {ref. }}$ For the case of the luffing crane, where the load height is directly affected by both $\beta$ and $l$, the reference values can be obtained from Eq.(3) by setting the sway variables, $\theta_{x}$ and $\theta_{y}$ to be zero and setting the load mass coordinates to the reference values. This gives

$$
\begin{gathered}
\emptyset_{\text {ref }}=C_{\emptyset}=\tan ^{-1}\left(\frac{C_{y}}{C_{X}}\right) \\
r_{r e f}=C_{r}=\sqrt{C_{x}^{2}+C_{y}^{2}} \text { or } 0 \text { (for luffing crane case) } \\
\beta_{\text {ref }}=C_{\beta}=\cos ^{-1}\left(\frac{C_{x}}{\left(r_{r e f}+r_{0}\right) \cos \emptyset_{r e f}}\right) \text { or } 0 \text { (for gantry crane) } \\
l_{\text {ref }}=C_{l}=-z_{\text {ref }}+\left(r_{r e f}+r_{0}\right) \sin \beta_{r e f}
\end{gathered}
$$

In the control system shown in Fig.4, $W B C$ is applied only to the $x$ and $y$ motions of the suspension point, whereas the hoisting length $l$ is directly controlled to follow the calculated reference values from Eq.(7).

The values of mechanical impedances, $Z_{x}$ and $Z_{y}$, in Eq.(1), were set to

$$
Z=a \cdot m \sqrt{\frac{g}{l_{0}}}
$$

where $m$ is the load mass and $a$ is a tuning parameter which can be set to unity initially. The choice of impedance values is not critical. For rest to rest manoeuvres, the initial linear momentum of the load is zero, and the control law in Eqs.(1) and (2) ensures that the final linear momentum is also zero, regardless of the choice of a finite value for $Z$. Furthermore, any finite value of $Z$ will also ensure energy absorption and so stability, throughout a manoeuvre and at arrival at the target 
position. The particular value then affects only the rate of energy absorption. It is not difficult to show that the role of $Z$ in the control law is to cause the actuator to behave as a viscous damper to the returning wave motion, that is, to cause the cable suspension point to have a velocity in a given direction which is proportional to the cable force in that direction. The value of $Z$ then determines the damping coefficient of the equivalent viscous damper. Eq.(8) with $a=1$ then gives about half critical damping for the pendular motion. The wave arriving back to the actuator then meets something close to a "matching impedance", to maximise absorption and minimise reflection.

The exact values of the components of the force at the top of the cable can be expressed as

$$
\begin{aligned}
& f_{x}=m\left[\ddot{x}_{T}+\ddot{l} \sin \theta_{x}+2 \dot{l} \dot{\theta}_{x} \cos \theta_{x}+\left(l+l_{0}\right) \ddot{\theta}_{x} \cos \theta_{x}-\left(l+l_{0}\right)\left(\dot{\theta}_{x}\right)^{2} \sin \theta_{x}\right] \\
& f_{y}=m\left[\ddot{y}_{T}-\ddot{l} \cos \theta_{x} \sin \theta_{y}+2 \dot{l}_{x} \sin \theta_{x} \sin \theta_{y}-2 \dot{i} \dot{\theta}_{y} \cos \theta_{x} \cos \theta_{y}+\left(l+l_{0}\right) \ddot{\theta}_{x} \sin \theta_{x} \sin \theta_{y}+\right. \\
& \left(l+l_{0}\right)\left(\dot{\theta}_{x}\right)^{2} \cos \theta_{x} \sin \theta_{y}+2\left(l+l_{0}\right) \dot{\theta}_{x} \dot{\theta}_{y} \sin \theta_{x} \cos \theta_{y}-\left(l+l_{0}\right) \ddot{\theta}_{y} \cos \theta_{x} \cos \theta_{y}+(l+ \\
& \left.\left.l_{0}\right)\left(\dot{\theta}_{y}\right)^{2} \cos \theta_{x} \sin \theta_{y}\right]
\end{aligned}
$$

For the control law only $f_{x}$ and $f_{y}$ are required. In a real crane they can be measured quite easily. Furthermore it is not necessary that their magnitudes be measured exactly. For rest-to-rest manoeuvres, their respective time integrals should return to zero, and their values should also return to zero, but exact tracking of the forces is not necessary. For example, it is sufficient to measure the cable angle and assume a constant (static) cable tension, $P=m \mathrm{~g}$, and simply set

$$
\begin{aligned}
& f_{x}=-P \theta_{x} \\
& f_{y}=P \theta_{y}
\end{aligned}
$$

thereby neglecting the dynamic loading effects, the trigonometrical nonlinearities, and possible errors in the value of the mass. Only when the sway has been reduced to zero will these forces, and their integrals, return to zero.

\section{Results}

\subsection{Rotary gantry crane}


In the following, the performance of the crane systems for some typical maneuvers is discussed to evaluate the efficiency of the developed control strategies. For example in the first test a gantry crane model undergoes a pure rotation in the $X Y$-plane, the constant length of cable and radial distance have been taken as $l_{0}=10 \mathrm{~m}$ and $r_{0}=20 \mathrm{~m}$ respectively. This maneuver is a constant-radius, slewing movement starting at $\varnothing=0^{\circ}$ to $\varnothing=132^{\circ}$ with no hoisting. The system's response to the slewing reference as the motion of the jib and the cable angles are shown in Fig.5.

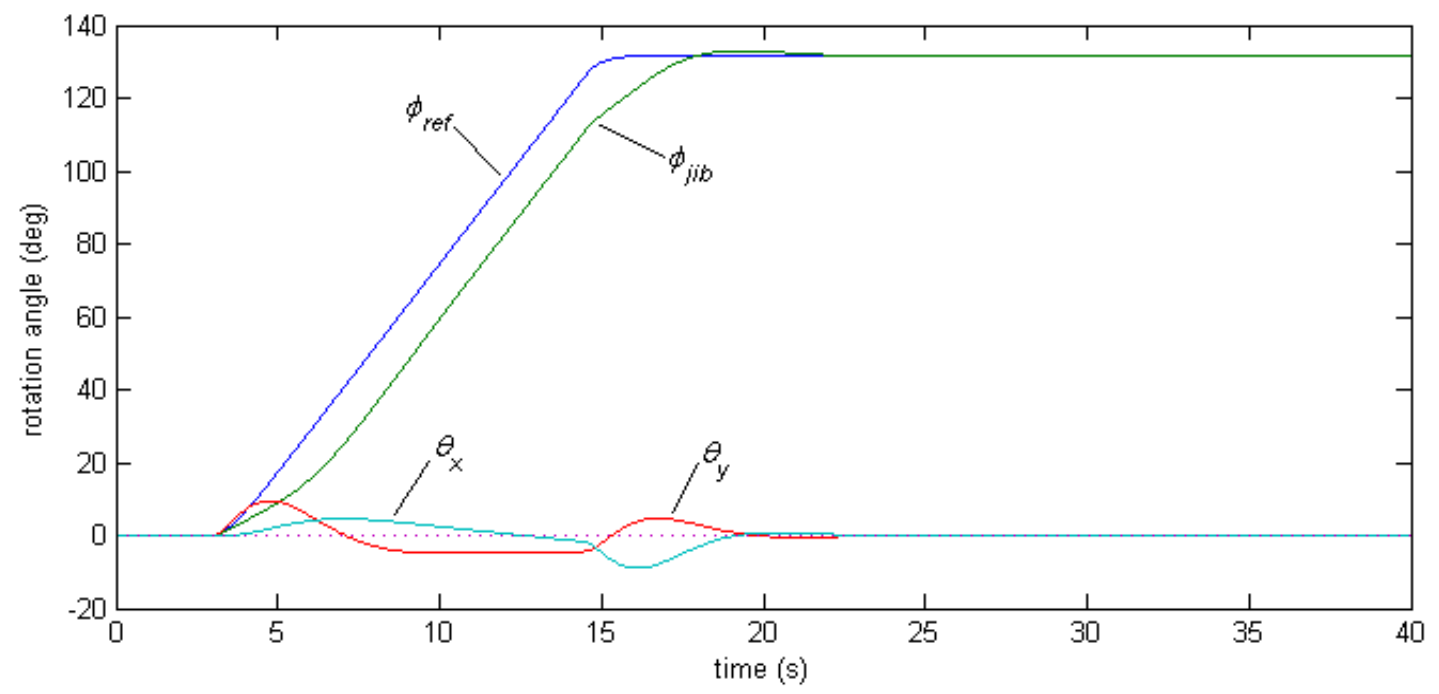

Fig. 5: Response of a gantry crane to a pure slewing reference: jib and cable angles

The jib is requested to slew through $132^{\circ}$ in about 10 seconds following a ramp reference input, with a 5-ton payload. Such a heavy-duty task in a relatively short time would normally involve a high level of residual swing as well as a delay in the response. However the jib settles at the target with negligible overshoot and little delay, while cable angles remain small throughout the transit. The cable angles during the main transit are resisting the centrifugal force on the load due to the slewing. The maximum cable deflection is less than 9 degrees, and happens only during the acceleration and deceleration phases when a larger angle is obviously appropriate and necessary to move the load. Throughout the manoeuvre WBC is working to try to keep the payload as close as possible to the reference track while actively damping the swinging action. Figure 6 shows how closely the payload tracks the target reference. A small trolley radial motion compensates for the centrifugal force to keep the load close to the target trajectory. 


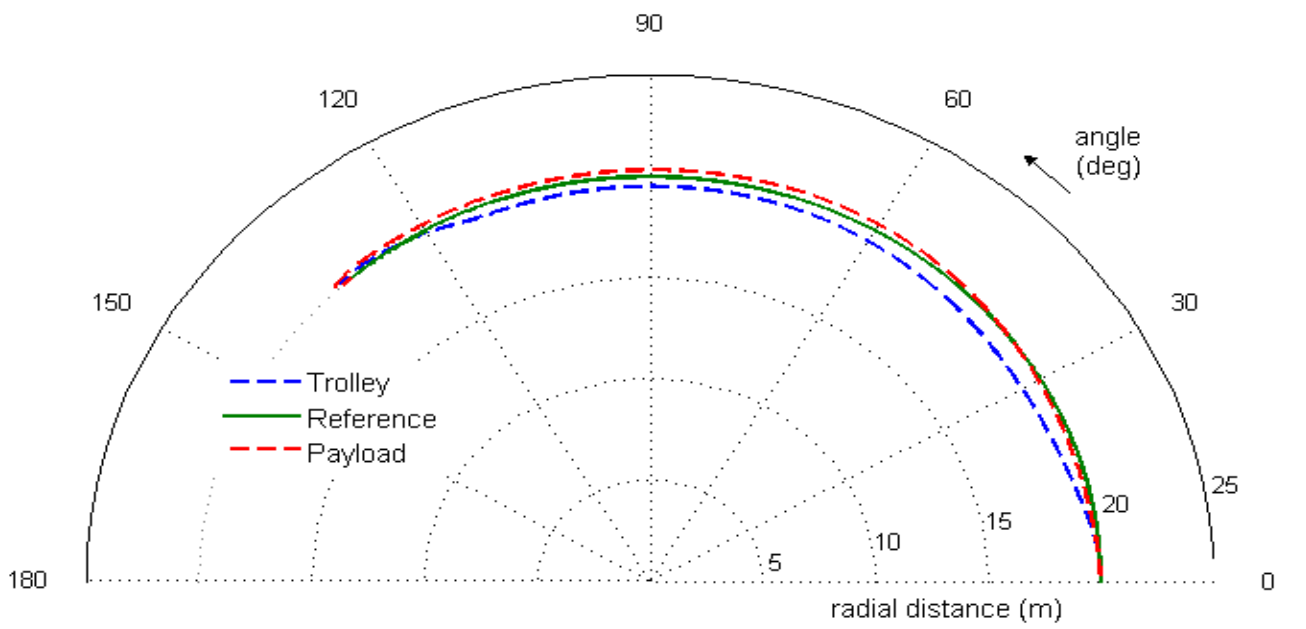

Fig. 6: Plan view of the slewing maneuver shown in Fig. 5 to show tracking.

The second test consists of a rotary crane combining slewing and radial motion over a longer time and with a complicated reference path, starting with $\emptyset_{\mathrm{jib}}=0^{\circ}, r_{0}=20 \mathrm{~m}$ and ending at $\emptyset_{\mathrm{jib}}=70^{\circ}, r=38 \mathrm{~m}$. The results in plan view are seen in Fig.7. The payload tracks the reference motion so closely that they are almost indistinguishable for most the manoeuvre, despite several sharp turns in the reference. The motion of the trolley to achieve this tracking is clearly visible. The sharp turns in the reference trajectory might be planned beforehand, for example to avoid known obstacles, or they might arise during the transit, in real-time, as a result of a reaction to an unexpected obstacle.

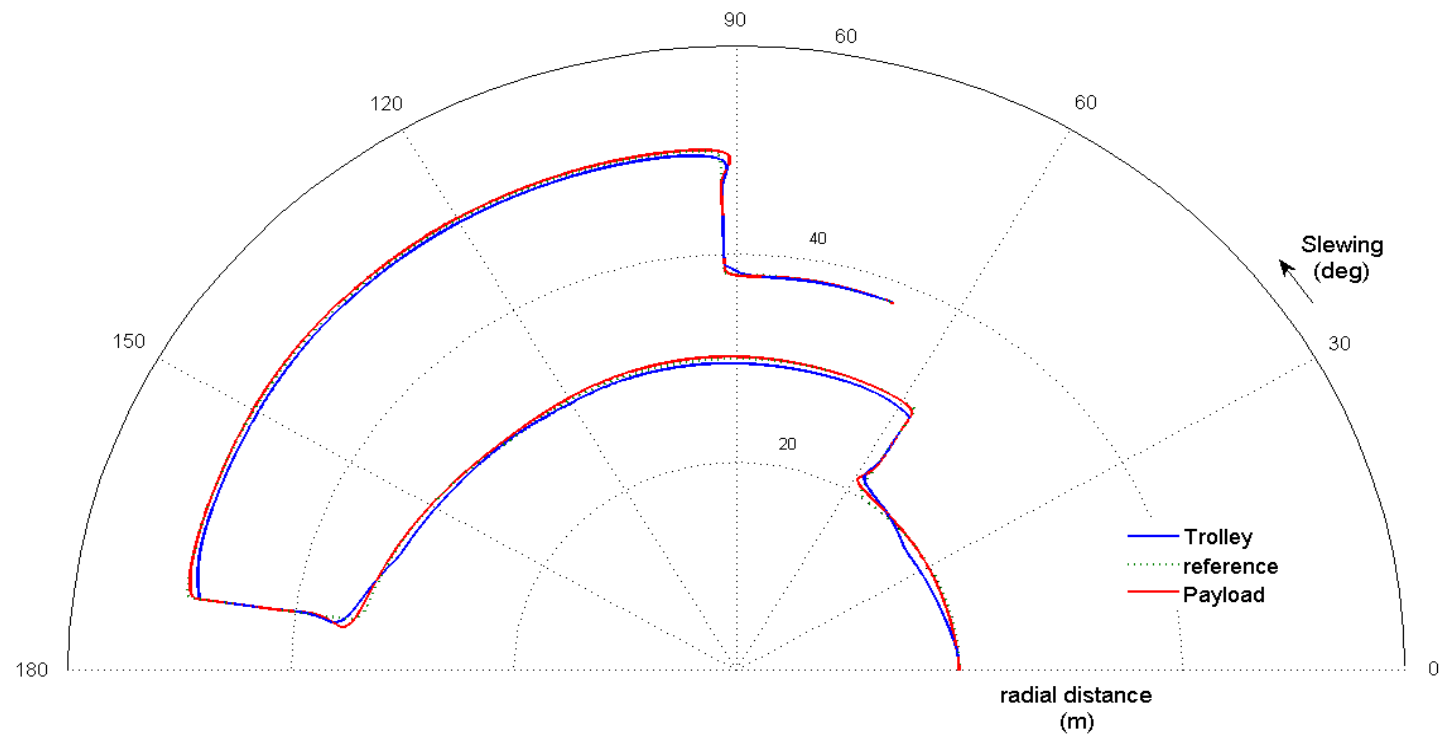

Fig. 7: Combined slewing and radial motion of a gantry crane under WBC: plan view

Figure 8 shows the radial and slewing motions against time for the same manoeuvre. The load 
motion is smooth, achieved by more complicated trolley and cable swing motions, indicating active sway control during the tracking.
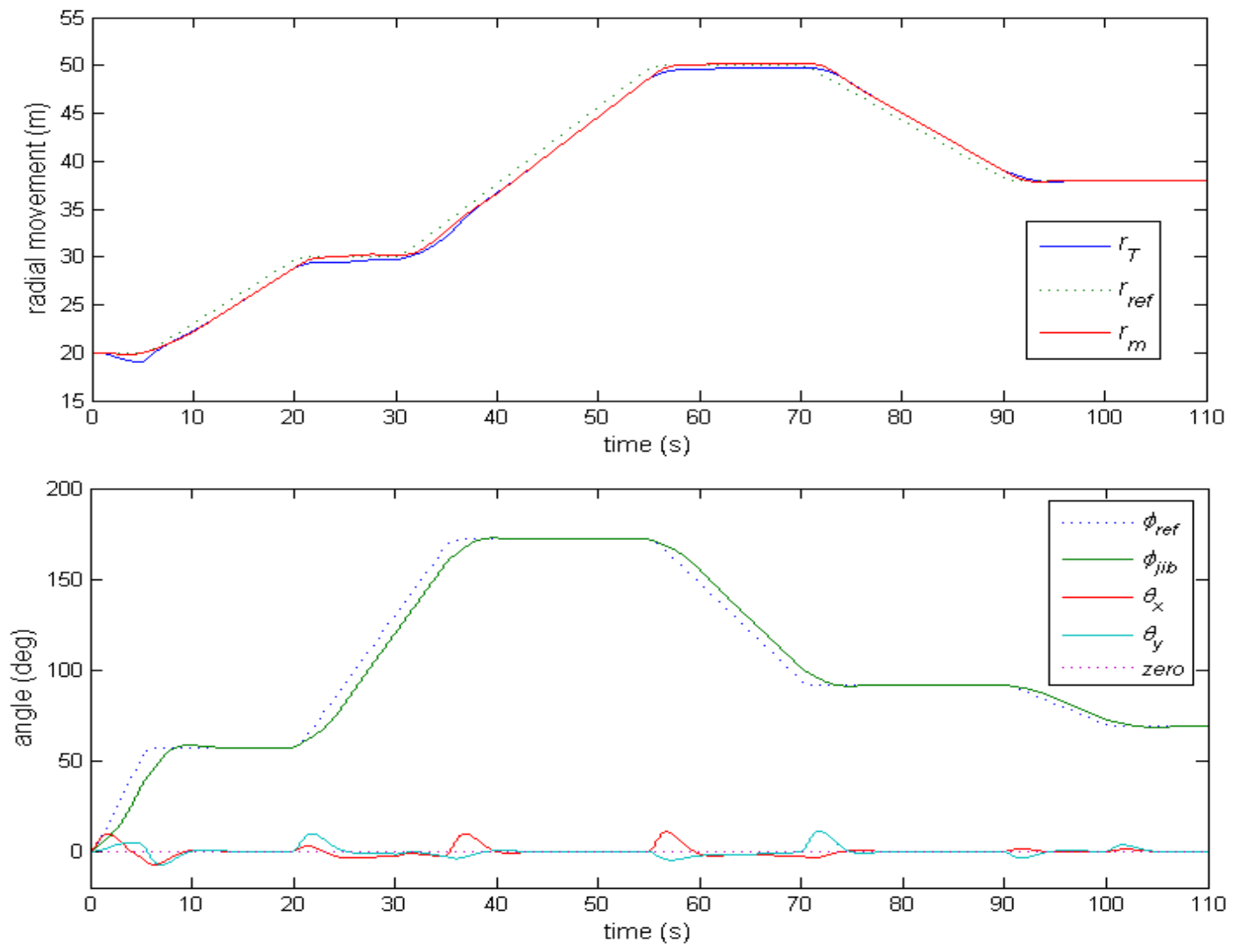

Fig. 8: Component motions over time for manoeuvre in Fig.7.

To illustrate the flexible dynamics more clearly, Fig.9 shows the response to the same reference inputs without wave-based (or other) control.

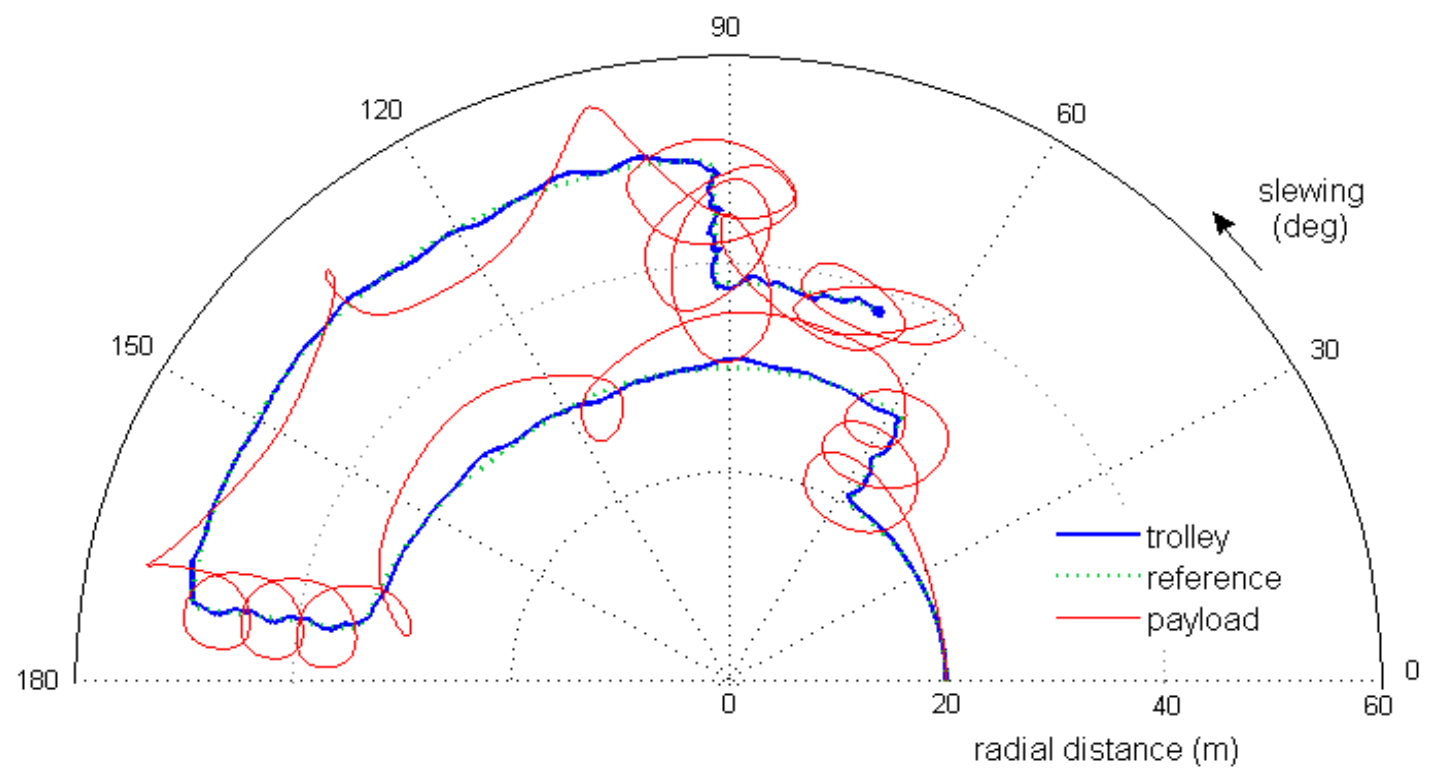


Fig. 9: Gantry crane without control undergoing the maneuver of Fig.7

\subsection{Luffing crane}

Sample results for a luffing crane are now presented, combining slewing and luffing motions simultaneously. Here $l_{0}=10 \mathrm{~m}$ and $r_{0}=20 \mathrm{~m}$, without hoisting. The captured behavior of the boom over time, and the reaction of the payload, shown by cable angles, can be seen in Fig. 10. The boom settles at the target angular position without any overshoot, although with a little time delay due to the returning wave's time lag.

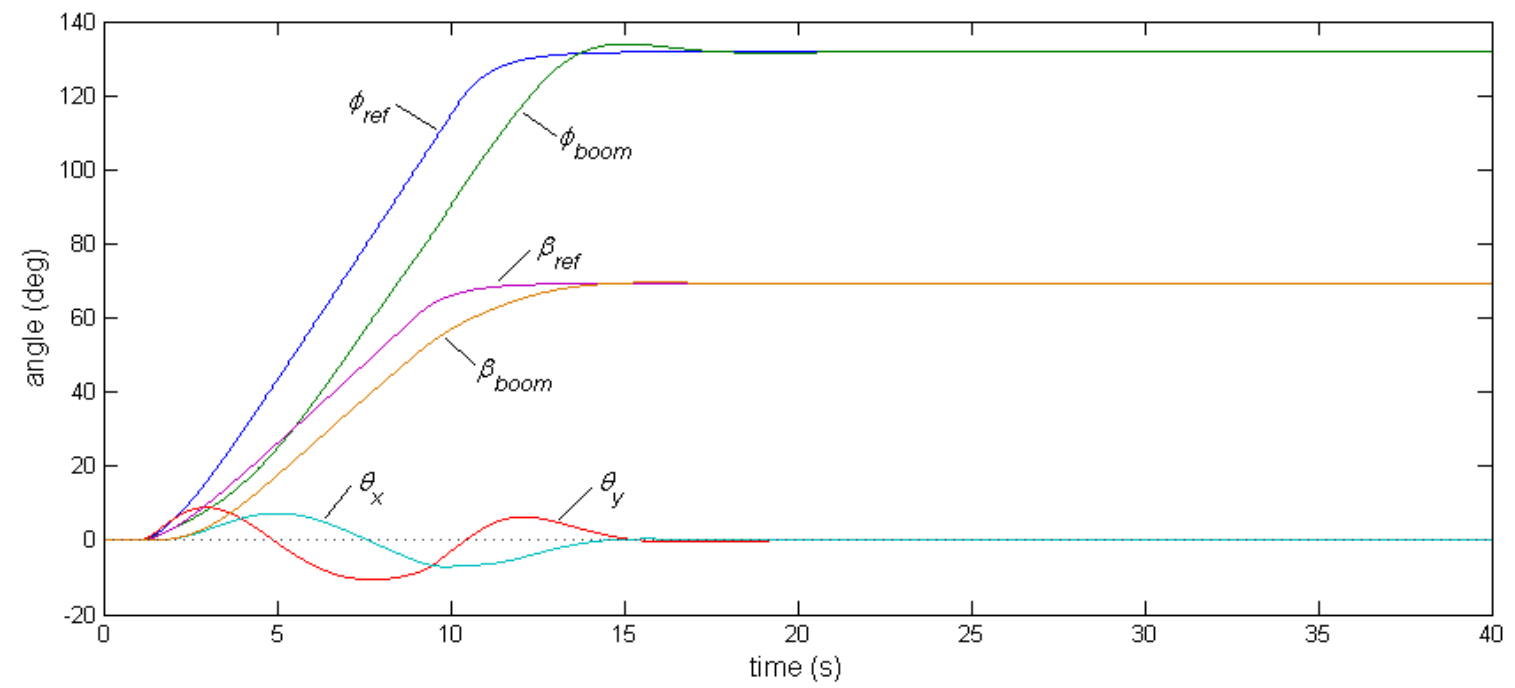

Fig. 10: combined slewing and luffing maneuver by a boom crane under WBC

Again centrifugal forces during slewing cause a radial displacement of the payload. Whereas the gantry crane under WBC used a suitable trolley displacement to keep the payload close to the target path (Fig. 6), the luffing crane must rely on an adjustment to the luffing angle to compensate for the radial swing. This can be seen in the plan view in Fig.11, which corresponds to the maneuver shown in Fig.10, and where the horizontal projection of the boom tip T (Fig.3) is plotted. 


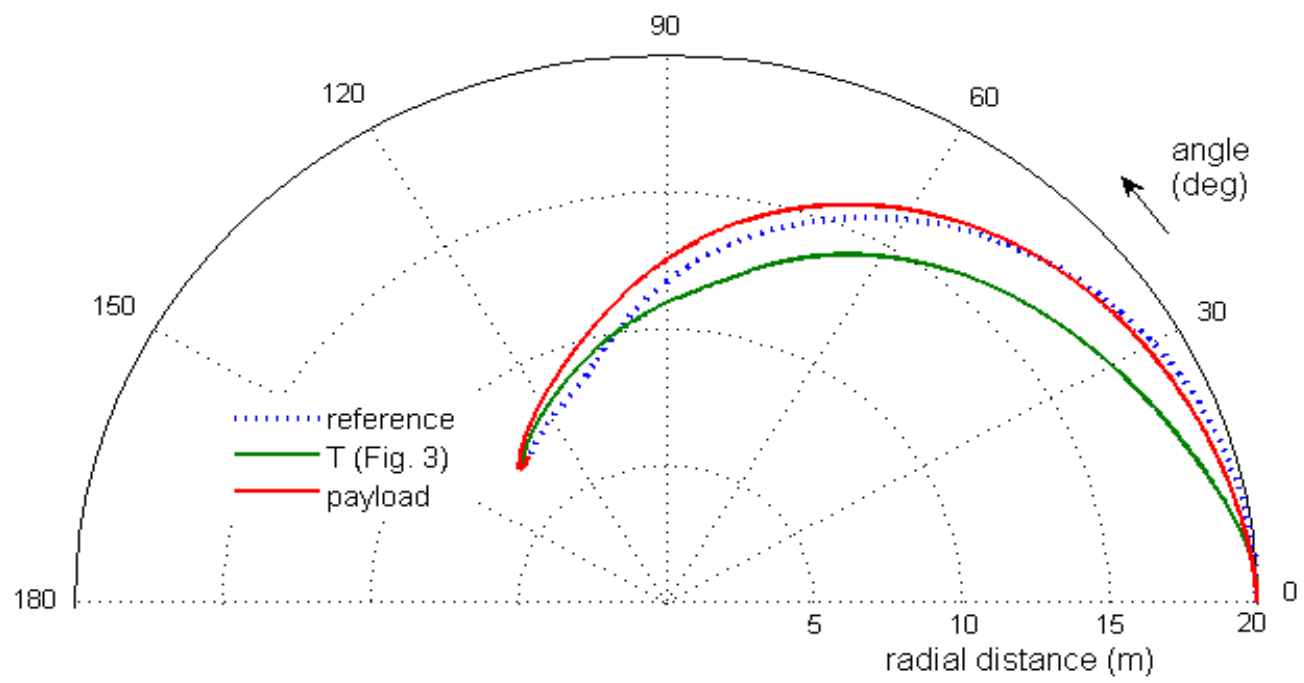

Fig. 11: Luffing crane, plan view, showing trajectory tracking for the same maneuver as in Fig. 10

Thus, for a boom crane attempting a purely slewing action, or constant radius load motion at a constant height, the controller compensates for the centrifugal force by lifting the boom. For example if the rotation in Fig. 6 , with a target radius of $20 \mathrm{~m}$, is carried out by a boom crane, then the radial position of the load in XY plane and the boom tip (T) are as shown in Fig. 12. The outreach of the boom is reduced by $1.2 \mathrm{~m}$ such that the load remains in the vicinity of the desired radius of $20 \mathrm{~m}$ with a deviation under $0.3 \mathrm{~m}$.

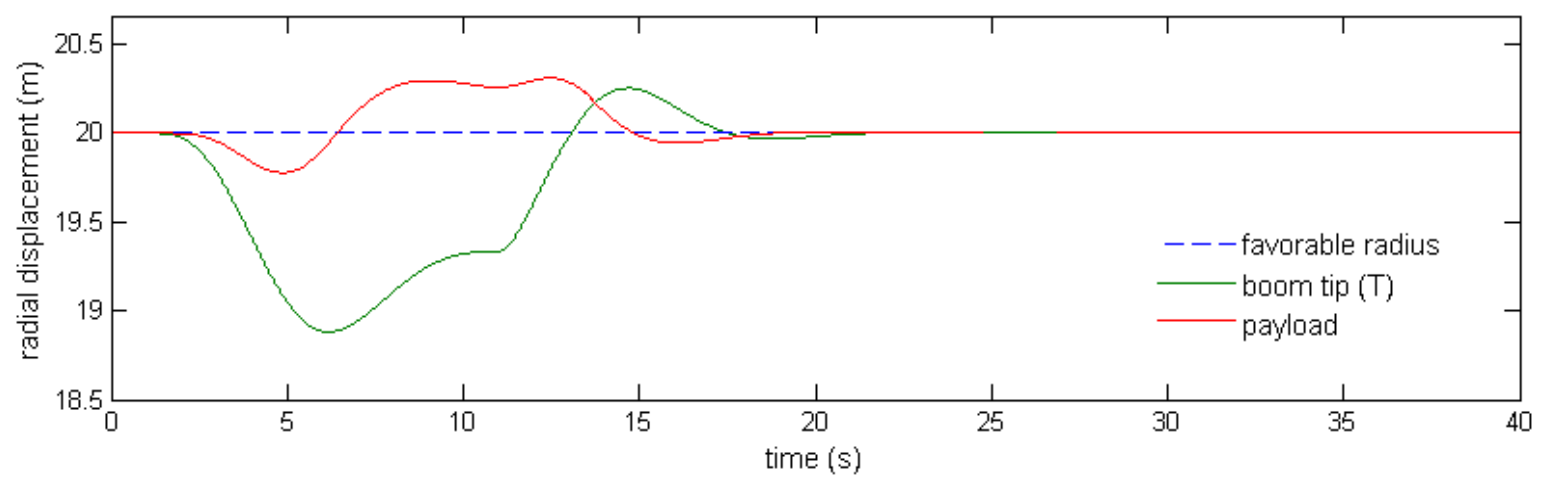

Fig. 12: Constant radius (20m) slew of a boom crane: load radius in XY plane and position error

\subsection{Hoisting}

Cable hoisting obviously changes the system dynamics considerably. One of the conclusions of this work, however, is that if the position and sway control is implemented using WBC in the horizontal plane, in perpendicular directions, $x$ and $y$, the design and implementation of the hoisting 
in the vertical or $z$-direction can be guided simply by the geometrical requirements dictated by the desired 3-D load trajectory. To illustrate this, two arbitrary 3-D manoeuvres are presented, one for a gantry crane, the other for a luffing crane.

For the gantry crane case, the mass is $5000 \mathrm{~kg}$, the initial length of the cable, $l_{0}=7 \mathrm{~m}$, and the reference jib length is $r_{0}=5 \mathrm{~m}$. The initial and final positions of the load are as follows:

\begin{tabular}{|c|c|}
\hline Initial load position & Target load position \\
\hline $\mathrm{x}_{0 \mathrm{~m}}=5$ (because $\left.\mathrm{r}_{0}=5 \mathrm{~m}\right)$ & $\mathrm{x}_{\mathrm{fm}}=8$ \\
\hline $\mathrm{y}_{\mathrm{m}}=0$ & $\mathrm{y}_{\mathrm{fm}}=4$ \\
\hline $\mathrm{z}_{\mathrm{m}}=-7$ (because $\left.l_{0}=7 \mathrm{~m}\right)$ & $\mathrm{z}_{\mathrm{fm}}=-1$ \\
\hline
\end{tabular}

The path from start to finish can be set arbitrarily. In the example they were designed as ramp-like transits in each direction, happening simultaneously. Figure 13 shows the results in 3-D. Figure 14 shows three planar views of the same maneuver, while Fig. 15 shows the radial motion over time. The example shows that, with hoisting, for some parts of the maneuver the load departs a little further from the target trajectory than before, but that the active swing control remains very good, and the load settles at the target position quickly and accurately.

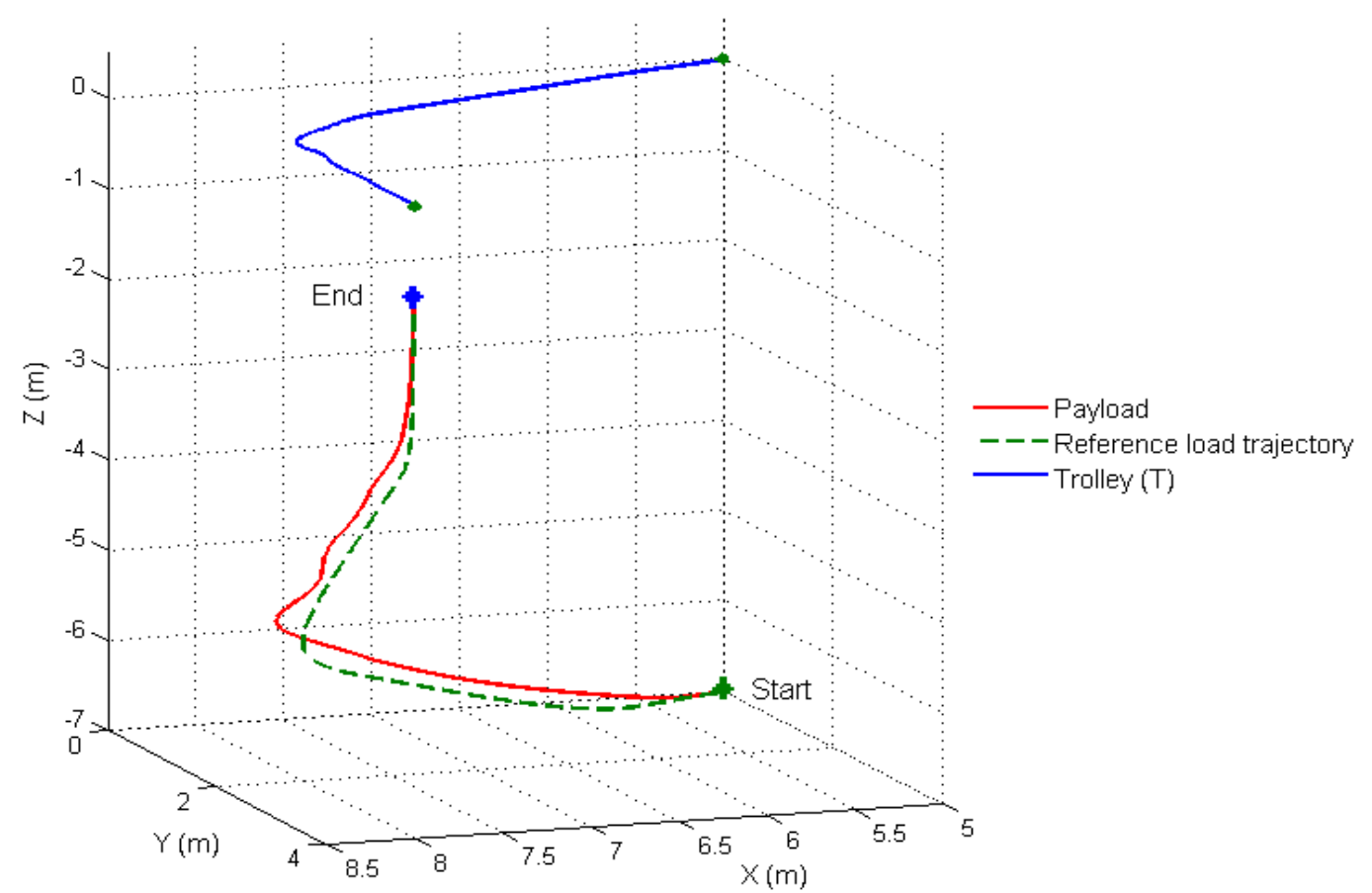

Fig. 13: Rotary gantry crane under WBC with hoisting, controlling $r, \emptyset \& l$ to follow a reference load trajectory. Curves show reference, actual load and trolley motions. 

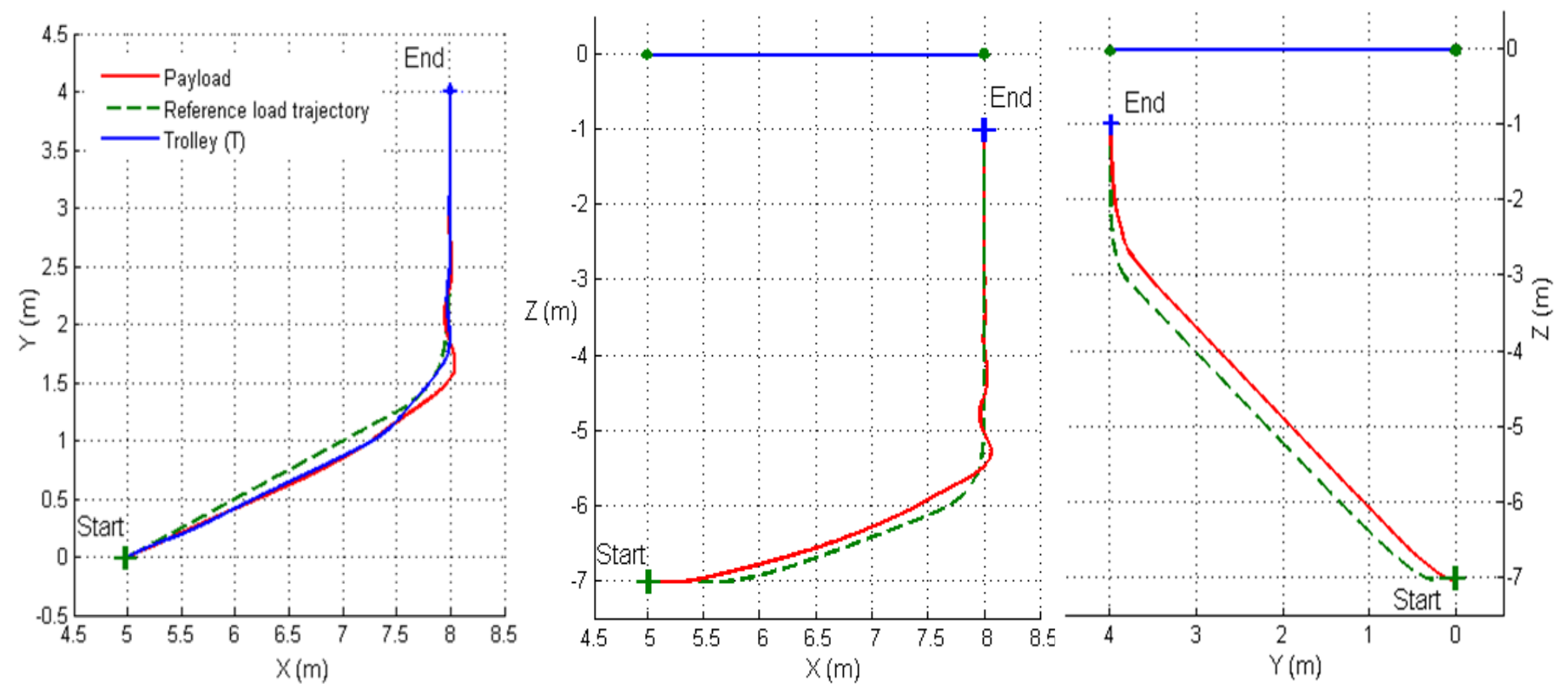

Fig.14: Plan view and two elevations (side views) of the 3-D manoeuvre of Fig.13.

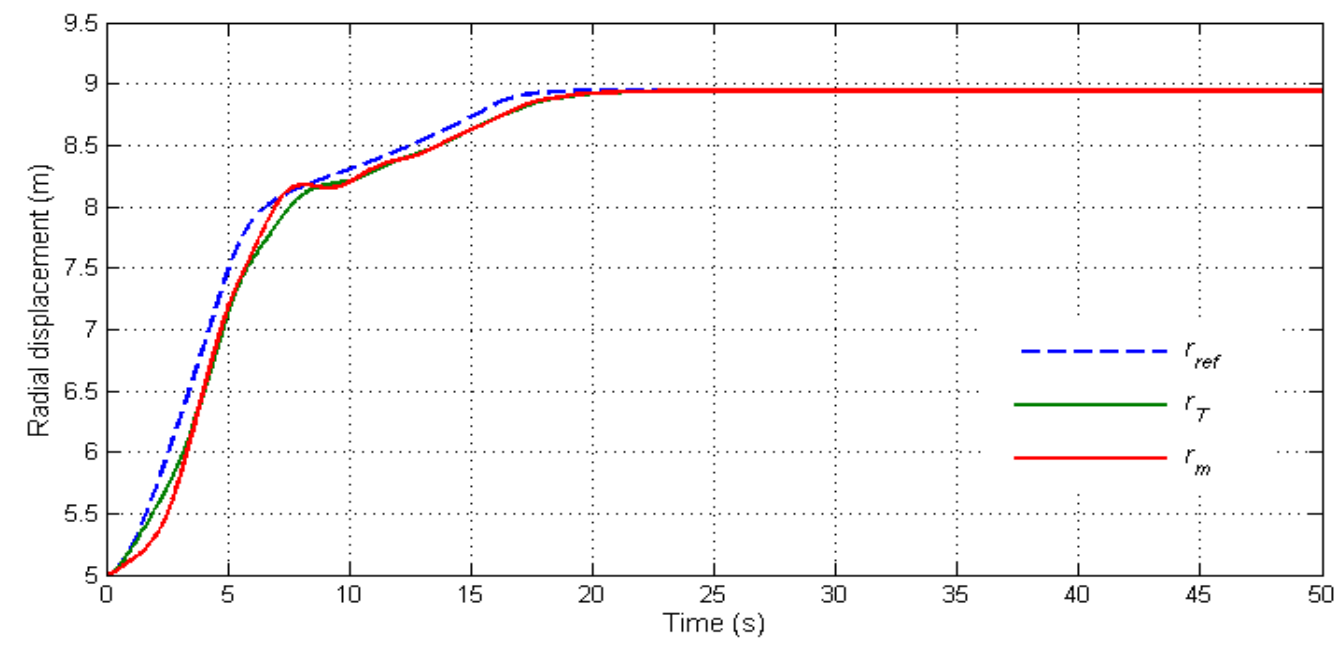

Fig.15: Radial motion over time for Figs. $13 \& 14$ : reference $\left(r_{r e f}\right)$, trolley $\left(r_{T}\right)$ and payload $\left(r_{m}\right)$

The final results are for a luffing crane with hoisting. The load mass is $m=5000 \mathrm{~kg}$, the constant boom length is $r_{0}=10 \mathrm{~m}$, and the cable initial or reference length is $l_{0}=3 \mathrm{~m}$. The initial load position and final target position were taken as

\begin{tabular}{|c|c|}
\hline Initial load position & Target load position \\
\hline $\mathrm{x}_{0 \mathrm{~m}}=10$ (because $\mathrm{r}_{0}=10 \mathrm{~m}$ ) & $\mathrm{x}_{\mathrm{fm}}=7$ \\
\hline $\mathrm{y}_{\mathrm{m}}=0$ & $\mathrm{y}_{\mathrm{fm}}=4$ \\
\hline $\mathrm{z}_{\mathrm{m}}=-3$ (because $l_{0}=3 \mathrm{~m}$ ) & $\mathrm{Z}_{\mathrm{fm}}=3$ \\
\hline
\end{tabular}


Again the 3-D transit assumed simultaneous ramp-like motions from initial to final positions in each variable, with challenging curves in the path. Figure 16 shows the 3-D trajectory, while Fig.17 shows the plan and two elevation views of the maneuver. The settling at the target position over time can be seen most clearly in Fig.18, for the vertical, z-motion, with the load traversing $6 \mathrm{~m}$ under shared luffing and hoisting actions. Again, in summary, while the tracking shows some departure from target path especially near the sharp curve, the swing control and final settling at target are very good.

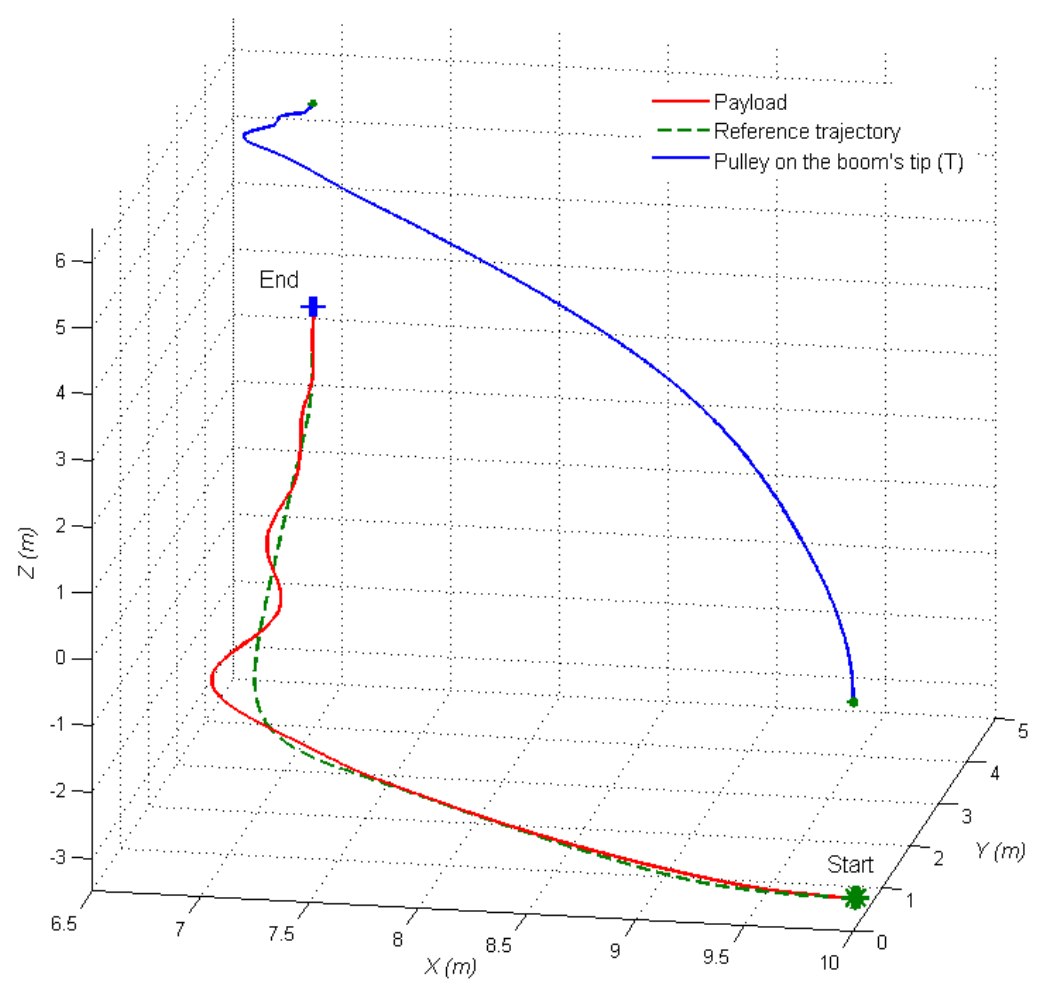

Fig.16: Luffing crane with hoisting under WBC, showing reference path, and motion of load \& boom tip, T. 


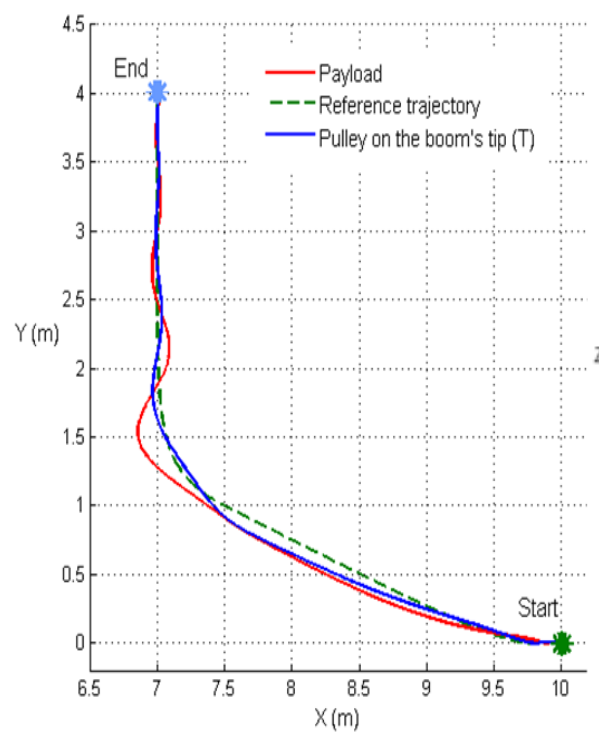

(a)

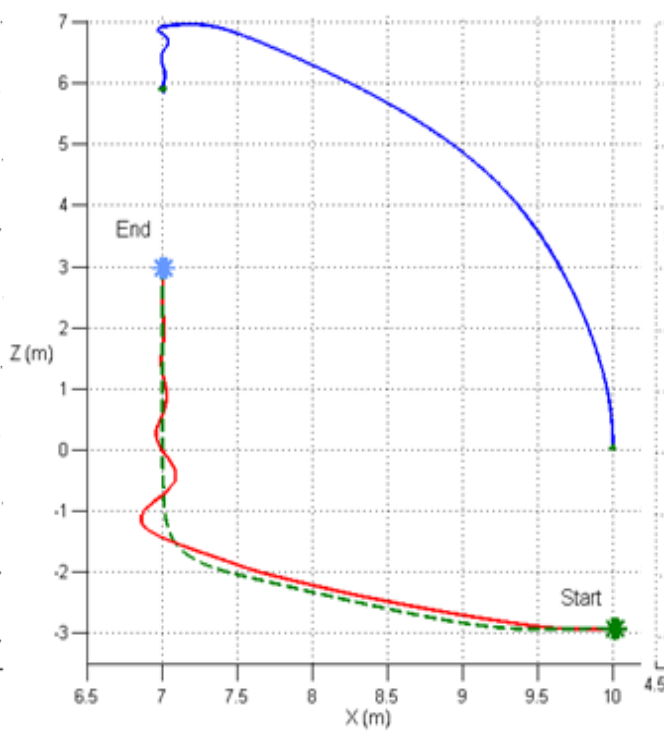

(b)

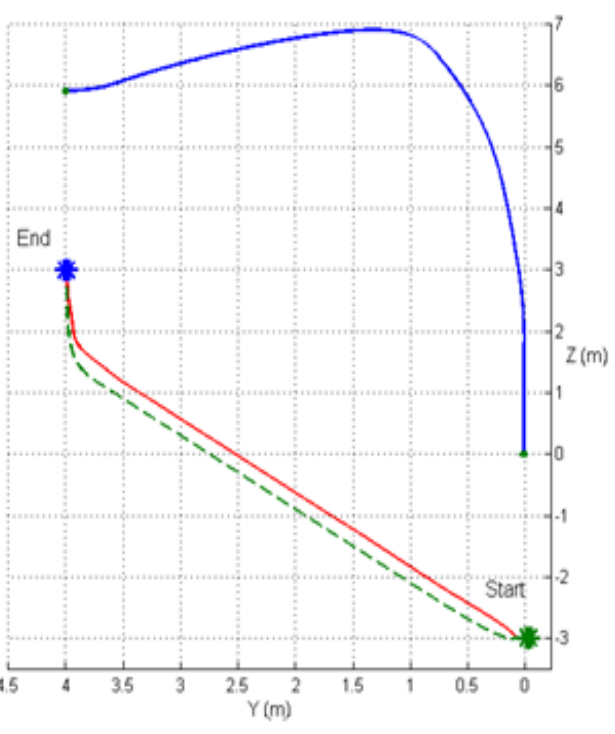

(c)

Fig.17: Plan and two elevation views of manoeuvre in Fig.16.

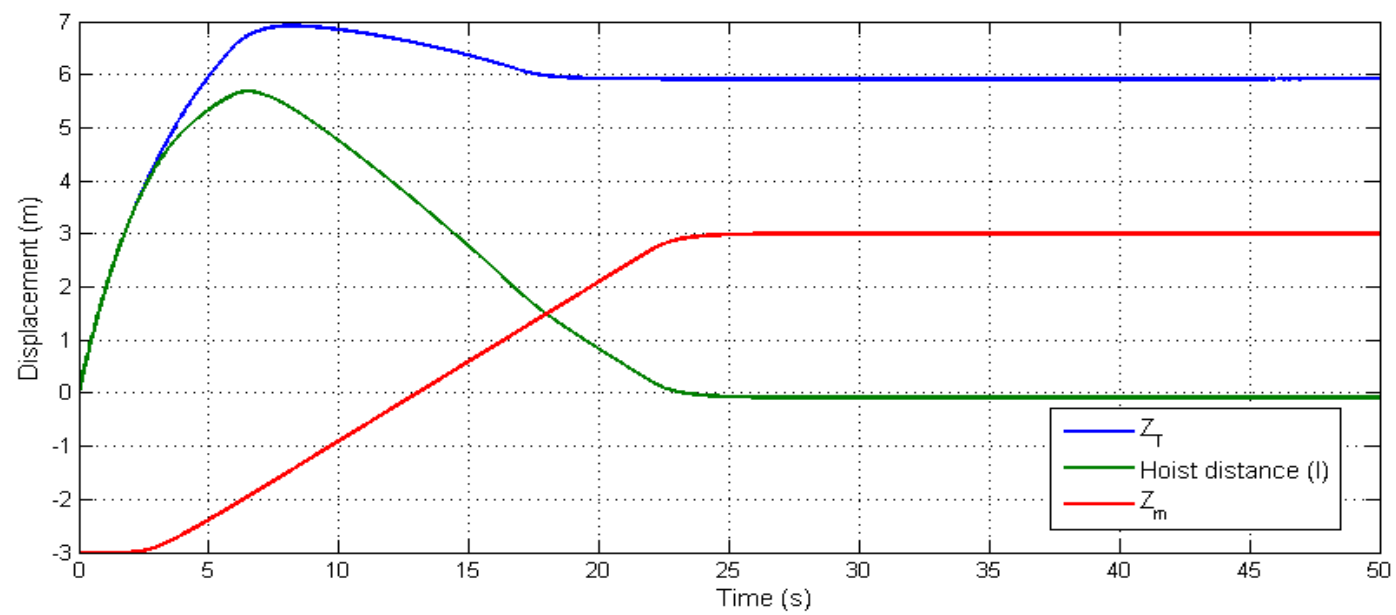

Fig.18: Vertical motion for same manoeuvre: boom tip, $\mathrm{Z}_{\mathrm{T}}$, hoisting, $l$ and load $\mathrm{Z}_{\mathrm{m}}$

The control system was tested for a wide range of system parameters and maneuvers (cable lengths, payload mass, and complexity of trajectories). Results very similar to those presented above were obtained. The only element of tuning of the controller is the choice of impedance value (Eq.(8)), and even that is not critical, in the sense that the system remains stable, with zero final error, over a wide range of values. Thus the parameter $a$ can be increased or decreased by, say, $50 \%$ without significant change in the main performance measures. If desired, for a given system and maneuver, it can be experimentally tuned, for example to reduce the overshoot at a sharp curve in a target 
trajectory, perhaps at the cost of a small increase in the time lag between the reference input and the load response.

To avoid lengthening this paper further, there are further attractive features of the control system which are not considered here, especially its robustness. For example, although the model developed above, and used to obtain most of the results, is somewhat idealised, the authors can report that the same wave-based control strategy still works very well for a real crane, or if a less idealised model is used to test the controller. For example, though not presented in this paper, the idealised hoisting motor model was given realistic dynamics, with inertia and friction, so that the actual cable length did not immediately follow the calculated (reference) length, $C_{l}$. For realistic motor dynamics there was negligible deterioration in performance. This success is because the wave measurements are based not on the calculated actuator inputs, but on the outputs actually achieved by the crane's dynamics, as measured at the interface. Similarly, in other work, when a load with more complex dynamics than a point mass was incorporated, the same control strategy worked well. The changed

dynamics cause changes in the measured motion returning to the measurement point at the actuator, and then the control automatically adjusts to absorb it, whatever form it takes. It is as if the controller has real-time system identification built in. The robustness regarding the value of the impedance can be understood as follows. If for any reason the absorption of the return wave is not optimal, and absorbs say only $90 \%$, the $10 \%$ reflected wave is re-launched into the system, but quickly finds its way back to the actuator, where $90 \%$ of this $10 \%$ is absorbed, and then the process repeats until all is absorbed. So the system still does a very good absorption job during the transit and settles at target very quickly.

\section{Summary and Conclusion}

In this paper wave-based control ideas were extended to the problem of automatic control of the load trajectory through 3-D space for a wide class of cranes having rotating towers. A generic crane 
model was developed, of which rotary, horizontal-gantry cranes and luffing-arm cranes are special cases. Hoisting effects were also considered. The control ideas were tested on the model. As in other work on wave-based control, directly controlled input variables were separately controlled in parallel to combine position control and active vibration (or swing) damping. It was found however that this separation strategy was fully successful and accurate only when the component motions were measured - and the wave-based control strategy implemented - in a Cartesian reference system, thereby uncoupling the motion components, at least for rest-to-rest manoevres at steady state. It was then necessary to transform between the cylindrical coordinates suitable for directly controlling the crane motions and the Cartersian coordinates in which WBC was implemented. This strategy was not difficult to implement and proved successful.

The main swing control was achieved using WBC on only the horizontal components of motion. This was effective in controlling the swing during path tracking, regardless of hoisting. This allowed the hoisting to be directly controlled as desired to achieve the target displacement over time in the vertical direction, taking luffing into account where appropriate, but without additional concerns about sway control.

The results show that the proposed control system ensures the swinging payload closely follows an arbitrary path in space, even when this path has sharp bends. The load position and swing are simultaneously controlled, even when the load is being accelerated and decelerated when cable sway angles are necessarily larger. All this is achieved with limited sensing: it is sufficient to know the position of the top of the cable and the cable angle in space at every instant to achieve real-time control. Although not presented in the paper, like in other applications of WBC, this strategy is also found to be robust to unknown or poorly measured parameters, to further complexities in the system dynamics, and to non-ideal behaviour of the crane actuation and hoisting. 


\section{References}

Abdel-Rahman EM and Nayfeh AH (2001) Feasibility of two-dimensional control for ship-mounted cranes. In DETC 2001 Proceedings of the ASME Design Engineering Technical Conferences, Pittsburgh, PA, DETC2001/VIB-21454.

Abdel-Rahman EM, Nayfeh AH and Masoud ZN (2003) Dynamics and Control of Cranes: A Review. J. Vib. Control 9(7): 863-908.

Blajer W and Kołodziejczyk K (2007) A DAE Formulation for the Dynamic Analysis and Control Design of Cranes Executing Prescribed Motions of Payloads. Multibody Dynamics: Computational Methods in Applied Sciences, Volume 4: 91-112.

Blajer W and Kołodziejczyk K (2011) Improved DAE formulation for inverse dynamics simulation of cranes. Multibody System Dynamics, Volume 25, Issue 2, pp: 131-143.

Gustafsson T (1995) Modelling and control of a rotary crane. In Proceedings of the $3^{\text {rd }}$ European Control Conference: ECC 95, Roma, Italy, pp: 3805-3810.

Matuško J, Ileš Š, Kolonić F, Lešić V (2015) Control of 3D tower crane based on tensor product model transformation with neural friction compensation, Asian Journal of Control, Volume 17, Issue 2.

Neupert J, Arnold E, Schneider K, and Sawodny O (2010) Tracking and anti-sway control for boom cranes. Control Engineering Practice, 18: 31-44.

O’Connor WJ (2003) A Gantry Crane Problem Solved. Journal of Dynamic Systems, Measurement, and Control, Vol. 125: 569-576.

O'Connor WJ (2007) Wave-Based Analysis and Control of Lump-Modelled Flexible Robots. Robotics, IEEE Transactions on, vol. 23: 342-352.

O'Connor W and Habibi H (2013) Gantry crane control of a double-pendulum, distributed-mass load, using mechanical wave concepts. Mech. Sci., 4: 251-261.

O’Connor WJ, Ramos F, McKeown DJ, and Feliu V (2009) Wave-based control of non-linear flexible mechanical systems. Nonlinear Dynamics, Springer, Vol. 57, No 1-2: 113-123.

Sakawa Y, Shindo Y, and Hashimoto Y (1981) Optimal control of a rotary crane. Journal of 
Optimization Theory and Applications 35(4): 535-557.

Takeuchi S, Fujikawa H, and Yamada S (1988) The application of fuzzy theory for a rotary crane control. In Proceedings of the International Conference on Industrial Electronics: IECON 88, Singapore, Vol. 2: 415-420.

Todd MD, Vohra ST, and Leban F (1997) Dynamical measurements of ship crane load pendulation. In Oceans 97 MTS/IEEE: Conference Proceedings, Hailfax, Canada, Vol. 2: 1230-1236.

Uchiyama N, Ouyang H, Sano S (2013) Simple rotary crane dynamics modeling and open-loop control for residual load sway suppression by only horizontal boom motion, Mechatronics, Volume 23, Issue 8 . 